(C) [2007] IEEE. Reprinted, with permission, from Zhao, C; Wang, Songnian; Qiu, Jie; Zhu, Jianguo; Guo, Youguang; Gong, Wz; Cao, Zj. 2007, 'Transient Simulation And Analysis For Saturated Core High Temperature Superconducting Fault Current Limiter', IEEE Transactions On Magnetics, Vol. 43, no. 4, pp. 1813-1816. This material is posted here with permission of the IEEE. Such permission of the IEEE does not in any way imply IEEE endorsement of any of the University of Technology, Sydney's products or services. Internal or personal use of this material is permitted. However, permission to reprint/republish this material for advertising or promotional purposes or for creating new collective works for resale or redistribution must be obtained from the IEEE by writing to pubs-permissions@ieee.org. By choosing to view this document, you agree to all provisions of the copyright laws protecting it. 


\title{
Transient Simulation and Analysis for Saturated Core High Temperature Superconducting Fault Current Limiter
}

\author{
Cuixia Zhao, Shuhong Wang, Jie Qiu, Jian Guo Zhu, Senior Member IEEE, Youguang Guo, Member \\ IEEE, Weizhi Gong, and Zhengjian Cao
}

\begin{abstract}
In this paper, the transient performance of a magnetic core fault current limiter (FCL) saturated by high temperature superconducting (HTS) DC bias winding is investigated by using both 3-dimensional (3D) field-circuit coupled simulation and magnetic circuit analysis. A high voltage is induced on the DC HTS winding during the fault current state. The induced voltage is computed and a short circuit coil for the reduction of induced voltage is studied. The numerical computations are verified by the experiment results on an FCL prototype.
\end{abstract}

Index Terms-Fault current limiter, high temperature superconductor, saturated core, field-circuit coupled simulation, magnetic circuit analysis.

\section{INTRODUCTION}

A S the results of the development of large-scale and complex implementation of power systems, the influence of the fault currents, such as short-circuit current and grounding current, has become one of important problems for electric appliances, e.g. the circuit breakers, transformers, and so on. All devices of the power system have to be designed to withstand the high mechanical and thermal stresses caused by the short circuit currents. There is a big demand for fault current limiters (FCLs), which has a negligible influence on a power system in normal condition, but can limit the current within a pre-defined value in fault condition.

High temperature superconducting fault current limiters (HTS FCL) are researched worldwide for their prospective commercial application in power system. Due to the superconductor and its special structure, the superconducting fault current limiter (SCFCL) will be a kind of innovative protection apparatus for high voltage grids [1]-[2]. It works automatically, further more, it offers many advantages: rapid reaction to fault current, low impedance in normal conditions, and large impedance during fault conditions. With the wide application of high temperature superconducting techniques,

Manuscript received Apr. 24, 2006.

C.X Zhao, S.H. Wang, and J. Qiu are with Xi'an Jiaotong University, China (phone: +86-29-82668630-106, e-mail: shwang@mail.xjtu.edu.cn).

J. G. Zhu, and Y.G. Guo are with University of Technology, Sydney, Australia (e-mail: joe@eng.uts.edu.au).

W.Z. Gong and Z.J. Cao are with Innopower Superconductor Cable Co., Ltd, Beijing, China (e-mail: gong_weizhi@innopower.com).
FCLs with diverse principles have been proposed, such as resistive, inductive, magnetic shielding and saturated core FCLs, etc. [3]-[5]. Most of them are based on the principle of the transition from the Superconducting to Normal conducting state (SN transition). However, the saturated core HTS FCL achieves the purpose of limiting short current not by using the SN transition, but by the nonlinear permeability of the magnetic core, which does not have the problem of recovery times. At the same time, the high temperature superconducting coils are supplied by DC source, hence do not have AC power loss, so more and more scholars have paid attention to this type of FCL.

There have been reports on analyzing the superconducting FCL by performing prototype experiment, as well as circuit model with $\Psi-i$ curve of FCL. The finite element analysis (FEA) considering $E-J$ characteristics of superconductor has also been developed in simulation of magnetic shielding type FCL [4]. In this paper, both methods of the equivalent magnetic circuit simulation and 3D transient magnetic field simulation are used to transiently simulate the saturated core FCL. To consider the nonlinearity of the magnetic core, in circuit simulation, an equivalent magnetization curve is used. This paper also utilizes the numerical simulation for the performance of HTS FCL by using 3D transient magnetic field computation directly coupled with circuit equations of AC windings, which is integrated in ANSYS. The results of the field and circuit variables are solved simultaneously. All of computed results and experimental results will be compared. The flux change of core will induce an undesirable high voltage in the DC coil, which may destroy the DC coil. In this paper, the induced voltage in the DC HTS bias winding is computed and analyzed in detail by using the transient FEA. A short-circuit coil will be investigated for limiting the induced AC voltage in HTS bias coil.

\section{TRANSIENT SimULATION AND ANALYSIS OF THE SATURATED CORE FCL}

\section{A. Analysis of the saturated core FCL by equivalent Magnetic circuit method}

1) Structure of saturated core FCL

The structure of the saturated magnetic core HTS FCL is shown in Fig. 1, which is composed of two magnetic cores and a superconducting DC bias coil. Although the permeability of 
the magnetic core is nonlinear, in order to analyze theoretically, the $\Psi-i$ curve of the both AC windings of FCL can be described approximately by five linear segments with different slopes, as shown in Fig. 2. Curve 1 illustrates the magnetization curve of single core without the DC bias, where $I_{c}$ is a critical saturated current, and $\Psi_{c}$ is the flux linkage. Curves 2 and 3 plot the magnetization curves of two cores, respectively, when a DC bias current $I_{d}$ is imposed. Curve 4 is the $\Psi-i$ curve of the saturated core FCL, which is achieved by summation of curve 2 and curve 3 .

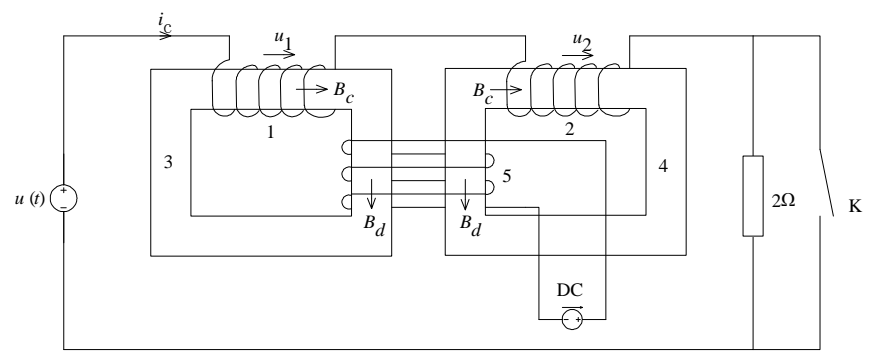

Fig. 1. Circuit for the current-limiting test 1, 2-AC windings; 3, 4-Magnetic cores; 5-Superconducting DC coils

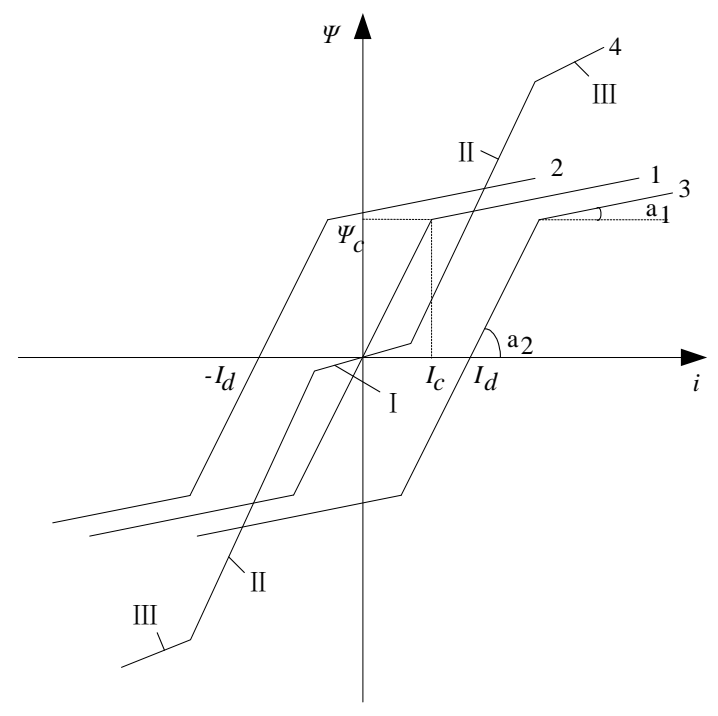

Fig. 2. $\Psi$-i curve of saturated core FCL

In the normal operation, both magnetic cores are driven into saturation by the DC current. The saturated core FCL works in line Segment I, so the impedance of the FCL is very low. When a short-circuit current occurs, the rapidly increasing AC current drives both magnetic cores out of saturation alternatively during one AC cycle, hence the saturated core FCL works in line Segment II, the impedance of the FCL becomes so large that the fault current is limited. However, if the AC current increases unceasingly, the magnetic cores will be saturated reversely, the FCL works in line Segment III, and will lose the capability of limiting current, due to the low impedance.

The DC bias winding for FCL is expected to use high temperature superconductors, which will drive the magnetic core into saturation with a low DC power supply.

\section{2) Magnetic circuit analysis}

The structure and working principle of the saturated core HTS FCL have been presented in Fig. 1, where the switch $\mathrm{K}$ is employed to simulate the short-circuit fault. The $B-H$ curve of the core is shown in Fig. 3.

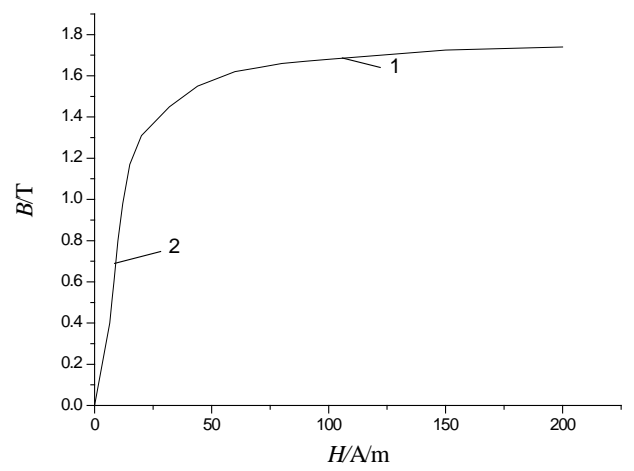

Fig. 3. $B$ - $H$ curve of magnetic core

In Fig. 3, we divide the $B-H$ curve into two segments labeled by numbers 1 and 2, respectively, and suppose that the turns of AC windings of each core and DC coil are $N_{\mathrm{c}}$ and $N_{\mathrm{d}}$, the mean length of magnetic circuit is $l$, and cross-sectional area of magnetic core is $A$.

According to Ampere's circuit law:

$N_{d} I_{d}-N_{c} i_{c}=H_{1} l$

$N_{d} I_{d}+N_{c} i_{c}=H_{2} l$

where, $I_{\mathrm{d}}$ and $i_{\mathrm{C}}$ are the currents of DC bias winding and AC winding, respectively, and $H_{1}$ and $H_{2}$ are magnetic field intensity of Core 1 and 2, respectively.

The derivatives of $\mathrm{H}_{1}$ and $\mathrm{H}_{2}$ can be written as

$$
\begin{aligned}
& \frac{d H_{1}}{d t}=-\frac{N_{c}}{l} \frac{d i_{c}}{d t} \\
& \frac{d H_{2}}{d t}=\frac{N_{c}}{l} \frac{d i_{c}}{d t}
\end{aligned}
$$

The relationship between magnetic flux density $B$ and $H$ is $B=\mu H$

The EMF of AC Windings 1 and 2 are shown as

$e_{1}=N_{c} A \frac{d B_{1}}{d t}=N_{c} A \mu_{1} \frac{d H_{1}}{d t}$

$=-\frac{N_{c}^{2} A \mu_{1}}{l} \frac{d i_{c}}{d t}$

$e_{2}=\frac{N_{c}^{2} A \mu_{2}}{l} \frac{d i_{c}}{d t}$

The induced voltage of AC Winding 1 can be derived as

$u_{1}=-e_{1}=\frac{N_{c}^{2} A \mu_{1}}{l} \frac{d i_{c}}{d t}$

Similarly, the induced voltage of AC Winding 2 is 
$u_{2}=-e_{2}=-\frac{N_{c}^{2} A \mu_{2}}{l} \frac{d i_{c}}{d t}$

Based on the Kirchhoff's voltage law, for the circuit in Fig. 1

$u(t)=u_{1}+u_{2}+i_{c} R$

Where, $R$ is the sum of load and the resistances of both AC windings.

Assume $\mu_{\mathrm{n}}$ is the permeability when the core is saturated. In the normal state

$\mu_{1}=\mu_{2}=\mu_{n}$

Considering (8)-(11):

$u(t)=2 \frac{N_{c}^{2} A \mu_{n}}{l} \frac{d i_{c}}{d t}+i_{c} R$

$=2 \frac{N_{c}^{2} A}{l} \frac{B}{H} \frac{d i_{c}}{d t}+i_{c} R$

$=2 \operatorname{tg} \alpha_{1} \frac{d i_{c}}{d t}+i_{c} R$

However, the permeability becomes $\mu_{f}$ when the core is not saturated. In the fault state

$\mu_{1}=\mu_{n}$

$\mu_{2}=\mu_{f}$

The two magnetic cores are saturated alternately, the voltage of both AC windings in series can be written as

$$
\begin{aligned}
& u(t)=\frac{N_{c}^{2} A \mu_{n}}{l} \frac{d i_{c}}{d t}+\frac{N_{c}^{2} A \mu_{f}}{l} \frac{d i_{c}}{d t}+i_{c} R \\
& =\left(\operatorname{tg} \alpha_{1}+\operatorname{tg} \alpha_{2}\right) \frac{d i_{c}}{d t}+i_{c} R
\end{aligned}
$$

3) Numerical result

According to all the above formulations, the dynamic current waveform is achieved and shown in Fig. 4, by using the equivalent magnetization curve and parameters of the saturated core FCL.

Fig. 5 is the measured current waveform under normal state and fault state. The investigation shows that the calculation results are in accordance with the experimental ones.

B. Simulation the saturated core FCL by $3 D$ transient magnetic field coupled with electric circuit

Because of the nonlinear magnetization (B-H) curve, this paper also employs the numerical simulation for the performance of the saturated core FCL by using 3D transient magnetic field computation directly coupled with circuit equations of AC windings, which is integrated in ANSYS. The transient magnetic field and circuit variables are solved simultaneously. The structure of a prototype saturated core FCL incorporated with DC HTS bias winding is shown in Fig. 6 . The $B-H$ curve of this magnetic core is shown in Fig. 3.

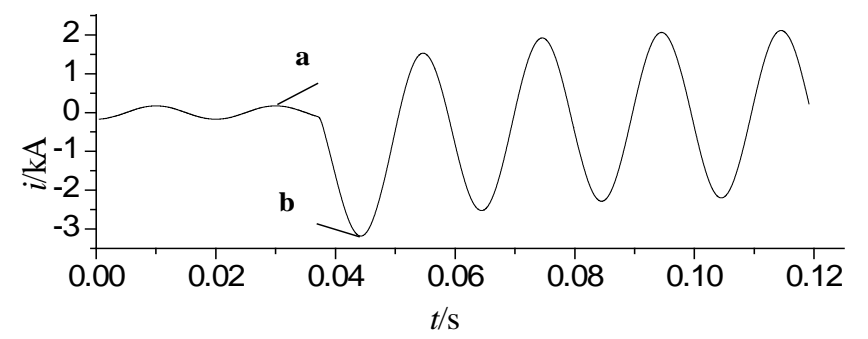

Fig. 4. Computational current by magnetic circuit 


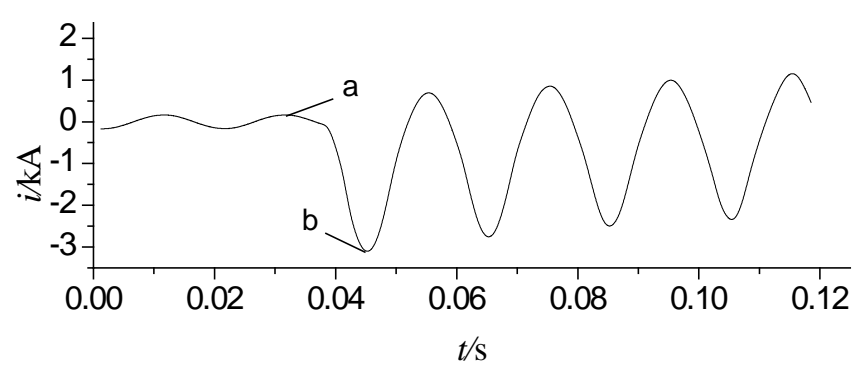

Fig. 7 Computational current by 3D transient FEA

\section{Comparison of the result between the experiment and simulation}

In this paper, we employed both methods to simulate the saturated core HTS FCL, and achieved the transient current, which are shown in Fig. 4 and 7 respectively. Table I and II list the results and relative errors of the simulated values and the measured value at the point "a" and the point "b", in Fig. 4,5 and 7. It shows good conformity in the results of both calculation and experiment.

According to Table II, the result of the numerical fieldcircuit coupled simulation is better than that of the magnetic circuit in high precision. Nevertheless, the latter possesses a better predominance in the aspect of the computing speed.

TABLE I

THe RESUlts AT POINTS “a” AND "b”

\begin{tabular}{ccc}
\hline \hline & $i_{\mathrm{a}}(\mathrm{A})$ & $i_{\mathrm{b}}(\mathrm{kA})$ \\
\hline Test & 158.0 & -3.09 \\
Circuit & 172.0 & -3.21 \\
FEA & 166.5 & -3.13 \\
\hline \hline
\end{tabular}

TABLE II

The Relative Error Between The Simulated Values And The

\begin{tabular}{cccc}
\multicolumn{4}{c}{ MEASURED VALUE } \\
\hline \hline Error & $\mathrm{a}(\%)$ & $\mathrm{b}(\%)$ & average(\%) \\
\hline Circuit & 8.86 & 3.88 & 6.37 \\
FEA & 5.38 & 1.29 & 3.36 \\
\hline \hline
\end{tabular}

\section{The High Voltage Problem of HTS Coils}

Fig. 8 shows the calculated voltage waveform of high temperature superconducting coils. It can be seen that the voltage is low in the normal condition. The influence for DC source can be negligible. However, in the fault condition, the high induced voltage of high temperature superconducting coils may destroy the superconducting coil and the DC source.

In order to decrease the induced AC voltage in the DC coil, a direct method is to reduce the turns of DC coils. However, this may lead to increasing current value of DC source. And a voltage-dividing device can be used in the DC circuit, for the purpose of dividing the voltage of DC source, which will consume more power.

In this paper, a short circuit coil as magnetic shielding is placed between the high temperature superconducting coils and the magnetic core, as shown in Fig. 6. According to Faraday's law of electromagnetic induction, this method will achieve the purpose of reducing the high induced voltage, in DC HTS bias coil. Comparing to both curves of fig. 8, it can be seen that the induced voltage with magnetic shielding is much lower than that without magnetic shielding. Thus, the short-circuit coil has an effect on reducing the induction of voltage.

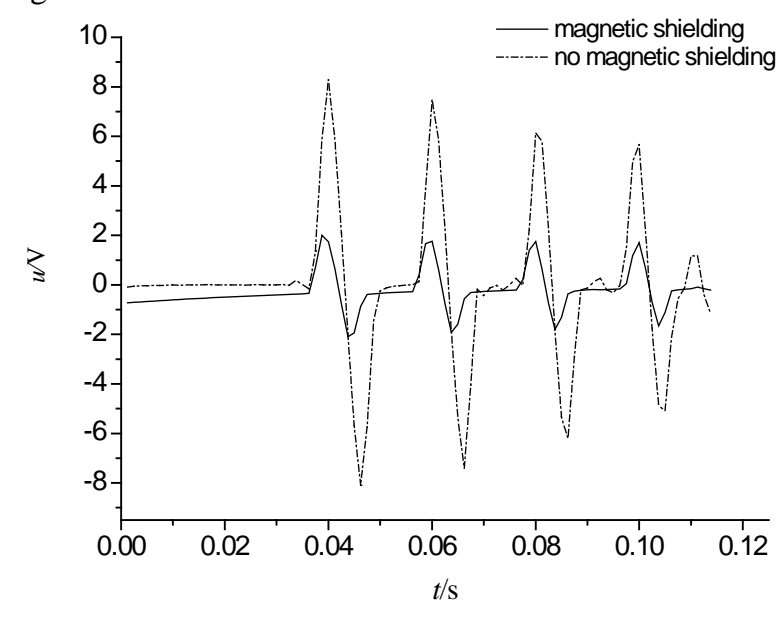

Fig. 8 Voltage waveform of HT superconducting coils

\section{CONCLUSION}

A saturated core FCL with HTS DC bias coil has been investigated by using the equivalent circuit method and 3D transient magnetic field simulation coupled with circuit. Good agreement between the computational and experimental characteristics confirms the accuracy of both methods. The saturated core FCL has a fast response to limit the fault current, because the superconductor has little transition time between superconducting state and normal conducting state. The high induced AC voltage in HTS DC bias coil, which may destroy the DC coil, has been simulated by applying FEA. A short circuit coil for reducing the voltage has been evaluated and discussed.

\section{REFERENCES}

[1] J.X. Jin, S.X. Dou, H.K. Liu and C. Grantham, "Preparation of high Tc Superconducting coils for consideration of their use in a prototype fault current limiter," IEEE Trans. Appl. Superconduct., vol. 5, pp.10511054, June 1995.

[2] H.J. Boenig and D.A. Paice, "Fault current limiter using a superconducting coil,” IEEE Trans. Magnetics, vol. 19,pp. 1051-1053, May 1983.

[3] M. Joo and T. K. Ko, "The analysis of the fault currents according to core saturation and fault angles in an inductive high- $\mathrm{T}_{\mathrm{C}}$ superconducting fault current limiter," IEEE Trans. Appl. Supercond., Vol. 6, pp. 62-67, June 1996.

[4] A. Ishiyama, J. Nakatsugawa, S. Noguchi, H. Kado, and M. Ichikawa, "Fundamental characteristic estimation based on finite element method for magnetic shielding type superconducting fault current limiter," Electrical Engineering in Japan, Vol. 134, pp. 17-27, 2001.

[5] V. Keilin, I. Kovalev, S. Kruglov, V. Stepanov, I. Shugaev, and V. Shcherbakov, "Model of HTS three-phase saturated core fault current limiter,” IEEE Trans. Appl. Supercond., Vol. 10, pp. 836-839, Mar. 2000. 